\title{
The Incomputable Alan Turing
}

\author{
S. Barry Cooper \\ School of Mathematics, University of Leeds \\ Leeds, LS2 9JT, UK \\ www.maths.leeds.ac.uk/ pmt6sbc \\ s.b.cooper@leeds.ac.uk
}

\begin{abstract}
The last century saw dramatic challenges to the Laplacian predictability which had underpinned scientific research for around $\mathbf{3 0 0}$ years. Basic to this was Alan Turing's 1936 discovery (along with Alonzo Church) of the existence of unsolvable problems. This paper focuses on incomputability as a powerful theme in Turing's work and personal life, and examines its role in his evolving concept of machine intelligence. It also traces some of the ways in which important new developments are anticipated by Turing's ideas in logic.

This paper is based on the talk given on 5th June 2004 at the conference at Manchester University marking the 50th anniversary of Alan Turing's death. It is published by the British Computer Society on http://www.bcs.org/ewics. It was submitted on 10th January, 2005; some minor amendments were made, and references added for publication, in March 2005.
\end{abstract}

Keywords: computability, machine intelligence, mathematical logic

\section{A BEGINNING ... AND AN END?}

Alan Turing's life and work had a peculiarly intimate relationship with each other. Thanks to Andrew Hodges' insightful biography [21], it is possible to trace many of the details of this, and to place it within the context of twentieth-century erosion of certainty.

"I am making a collection of experiments in the order I mean to do them in. I always seem to want to make things from the thing that is commonest in nature and with the least waste in energy."

So wrote (quoted by Hodges, p.19) the young Alan Turing in a letter home from boarding school in March 1925, setting out the essentials of a lifelong agenda - one whose shadowy outcome, with the very nature of predictability in question, could not have been foreseen. Here (wrenched out of context) is the closing paragraph (p.527) of Hodges' book:

"With so few messages from the unseen mind to work on, [Alan Turing's] inner code remains unbroken. According to his imitation principle, it is quite meaningless to speculate upon his unspoken thoughts. Wovon man nicht sprechen kann, darüber muss man schweigen. ${ }^{1}$ But Alan Turing could not possess the philosopher's detachment from life. It was, as the computer might put it, the unspeakable that left him speechless."

What had happened by the end was that Turing had played a key role in pushing forwards the boundaries of machine intelligence, had struggled with newly discovered theoretical and practical obstacles to computability, and humanly confronted the parallel realities of a world out of control. And just as it was hard for Turing to make sense of what he had played a role in revealing, we have to be cautious, fifty years later, in interpreting and simplifying Turing's own views.

\footnotetext{
${ }^{1}$ Usually translated as: "What we cannot speak about we must pass over in silence." - being the final sentence of Ludwig Wittgenstein's Tractatus Logico-Philosophicus.
} 
Some of the themes addressed in this article will be: Turing's investment in computability, incomputability, and in their practical dimensions; his work in logic, and the ever present real world connection; and the relevance today of the Turing approach to logic - and its anticipation of current research themes.

\title{
2. CONFRONTING THE INCOMPUTABILITY BARRIER - THE ALAN TURING WAY
}

Incomputability has been with us for much longer than has the precise sense the term today derives from computability theory. Who has not made plans based on best calculations, only to be surprised and thwarted by the unpredictability of the real world? Even the word itself has been with us for around four hundred years. The New Oxford Dictionary of English (1998) gives the meaning "unable to be calculated or estimated, origin early 17 th cent." In everyday usage, incomputability is a barrier to human activity - something we all experience in everyday life.

At this level, the commonest coping strategy is a cultural one - imitation. One adopts strategies learned from copying what works for other people. A more individual, and necessarily rational, one is that of reductionism and thought experiments. Here, complex phenomena are broken down into basic ones, the aim being solutions we recognise as being scientific. We can describe this as 'computing with awareness'.

For Alan Turing, strategy I was not a favoured option! For him, the experience of incomputability was an intimate one, his scientific and personal life inextricably intertwined. To this extent his mind became a ready-made laboratory for exploring the computable/incomputable interface. Here is how his older brother John (Hodges, p.33) describes, around 1928, Alan's brave approach to making sense of the world:

"You could take a safe bet that if you ventured on some self-evident proposition, as for example that the earth was round, Alan would produce a great deal of incontrovertible evidence to prove that it was almost certainly flat, ovular, or much the same shape as a Siamese cat which had been boiled for fifteen minutes at a temperature of one thousand degrees Centigrade."

\section{3. ... AND FINDING THIS BARRIER ALL-TOO-REAL}

On Thursday 13 February 1930, Turing's school friend Christopher Morcom died. This unexpected blow to the young Alan Turing (see Hodges) was a seismic shattering of his personal Laplacian universe. Alan tried to make sense of what had happened, writing to his best friend's mother, Mrs. Morcom, around 1932 (quoted in Hodges, pp. 63-64):

\begin{abstract}
"It used to be supposed in Science that if everything was known about the Universe at any particular moment then we can predict what it will be through all the future. ... More modern science however has come to the conclusion that when we are dealing with atoms and electrons we are quite unable to know the exact state of them ... The conception then of being able to know the exact state of the universe then really must break down on the small scale. This means then that the theory which held that as eclipses etc. are predestined so were all our actions breaks down too. We have a will which is able to determine the action of .... atoms... in ... the brain,... ... matter is meaningless in the absence of spirit ... as regards the actual connection between spirit and body I consider that the body by reason of being a living body can 'attract' and hold on to a 'spirit' ... The body provides something for the spirit to look after and use."
\end{abstract}

It is worth looking more closely at this for what it tells us about Turing's thinking at this time. One is struck by a fluidity of thought, anticipating current concerns (and confusions!) about computability. There are tentative steps towards a view of the Universe as information. There is the awareness of, and interest in, the then very recent discovery of quantum uncertainty. For the young Turing (familiar with Eddington [11], for example) there is a very contemporary resonance between the personal and the scientific. Human spirit becomes admissible, embodying special information 
content, from which it gets purpose and persistence. And there are those last three wonderful clauses, full of a very contemporary sense of the mystery and significance of the relationship between different levels of complexity, and even a premonition of the underlying phenomenon of mathematical definability detected in recent explanations. And of the necessity of incomputability. Notice that at this point the mind is not seen as mechanical.

Is the above quotation little more than the unformed and naive writings of a young man getting through an emotionally stressful part of his life? One can so dismiss them. But only three or four years later, Turing was working on one of his most admired and influential scientific papers.

\section{THAT REMARKABLE 1936 PAPER}

Turing [37] did not actually appear until January 1937. Typically, Turing starts with a more basic question than that asked by other authors. Not "What is a computable function?". But (p.249):

'The real question at issue is "What are the possible processes which can be carried out in computing a [real] number?",

For a full commentary on this paper and its historical context, see Robin Gandy [18] - this is essential reading, of course, from Turing's ex-student and friend. Here is a familiar picture (taken from Cooper [2]) of Turing's hardware, all computational subtlety residing in the program.

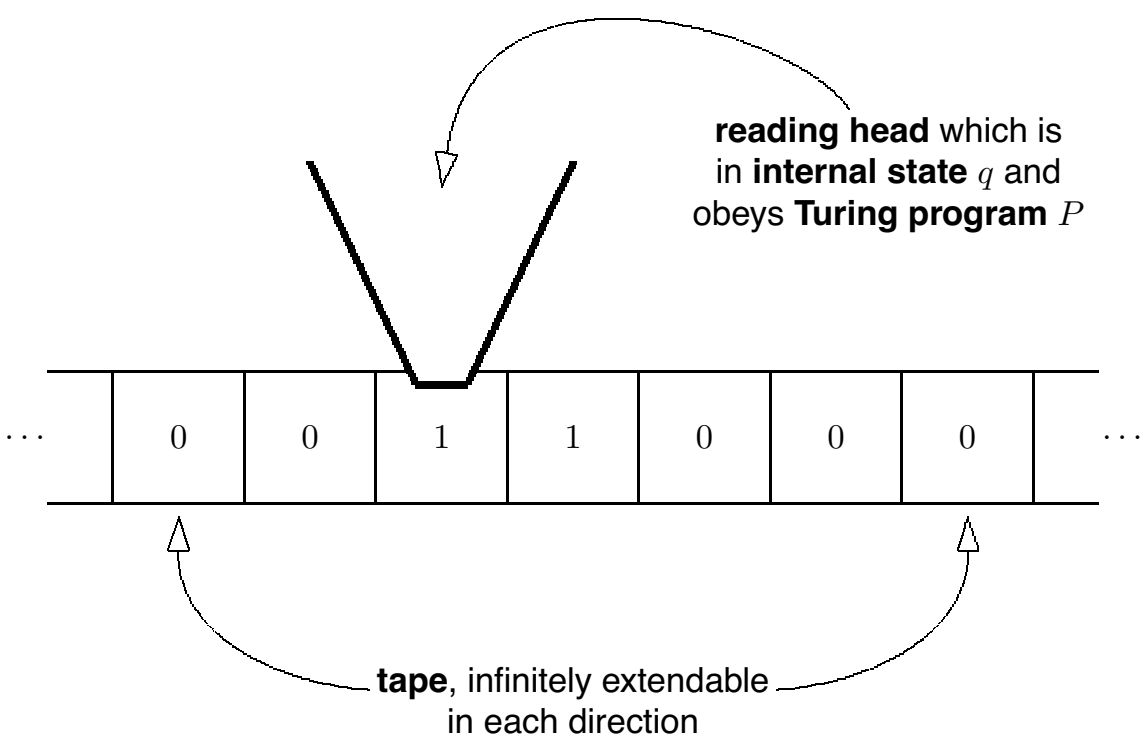

The Turing machine may not have been the first general notion of computability - Post even had a machine notion of computability (not published) which was very similar to Turing's. But it was the most convincingly formulated and presented. It won over Gödel (see John Dawson [10], pp. 101-102). Gödel's long-time friend Hao Wang provides ([45], p.96), the final word on Kurt Gödel and Church's thesis:

"Over the years G habitually credited A. M. Turing's paper of 1936 as the definitive work in capturing the intuitive concept [of computability], and did not mention Church or E. Post in this connection. He must have felt that Turing was the only one who gave persuasive arguments to show the adequacy of the precise concept ... In particular, he had probably been aware of the arguments offered by Church for his 'thesis' and decided that they were inadequate. It is clear that $G$ and Turing (1912-1954) had great admiration for each other, ..." 
Over the years, of course, the proved equivalences between the diverse models of computability gave plenty of evidence that the Church-Turing Thesis (as it is now called) is essentially correct. But it is the persuasiveness of Turing's original argument which still has more powerful intuitive content for us.

Turing's paper is characteristically basic in other ways. For instance, it also takes account of the scientist's need to describe the world in terms of real numbers. Turing's 'computable real numbers' embodied concessions that the soon-to-become dominant recursive function theoretic framework clearly did not.

\section{THE UNIVERSAL MACHINE AND THE COMPUTER}

Not everything in the paper was so accessible. The student who imagines it was simply Turing's invention of the Turing machine which anticipated the computer is missing the main point of his paper. Turing's idea was to Gödel number (or code) programs, while building a universal Turing machine $U$ capable of decoding and implementing any coded program.

As Martin Davis [8] points out - at a stroke, Turing anticipated:

- Interpretive programs - in that $U$ unscrambles codes into implementable information (quintuples),

- The Stored Program Computer - whereby the distinction between program and data evaporates,

- The versatility of today's computers, with hardware solutions replaced by equivalent software.

It is clear from Turing's paper that his universal machine is only universal in a very specific sense. Turing's machines - and the $U$ which simulates them - were framed in terms of the computing capabilities of a human clerk. Those of machines in general remained to be fully pinned down. Turing's later involvement with physical computability was to bring to the surface some contemporary themes still occupying those seeking to mathematically model how the real world computes.

What is also clear, is that the universal Turing machine provides an accurate basic model of how the computer on our desk computes. From the perspective of the pure mathematician, Turing did invent the computer. You can write down the program for $U$ and implement it in a full range of contexts, admittedly in too inefficient a way for it to have any practical use! Turing's name and reputation still has power to enrage the engineer concerned with implementing that abstract idea. Turing's own involvement in the building of those first computers just makes his role even more irritating to those who neither recognise, nor want to recognise, the mathematical structures which constrain their daily lives.

Turing's best-known early influence on computer design was through John von Neumann, particularly via the latter's EDVAC report (1945). Although von Neumann does not specifically acknowledge the significance of Turing there, he later gave proper credit in his Hixon Symposium lecture, in September 1948 (see [44]). Understanding of Turing's key role in the design of early computing machinery, such as the 'Colossi' (circa 1944), during the Second World War, is to a great extent lost through Churchill's deliberate obliteration of physical evidence of the Bletchley Park story.

But relevant for us today, as Robin Gandy [19] tells us (p.17) in his Preface to the 1936-37 papers in the Collected Works of A.M. Turing: Mathematical Logic:

"Turing machines ... still provide the standard setting for the definition of the complexity of computation in terms of bounds on time and space; 
- together with the neural nets of McCulloch and Pitts they provided the foundations of the theory of automata;

- together with the generated sets of Post [1943] they provided the foundation for the theory of formal grammars."

\section{HILBERT'S PROGRAMME, AND NATURAL EXAMPLES OF INCOMPUTABLE OBJECTS}

Turing's paper was not immediately useful, of course. Its practical benefits lay still some years in the future. But it did relate to a grand scientific enterprise traceable back to Newton and beyond, that of capturing the algorithmic content of the natural world. As Albert Einstein [12] said (p.54):

"When we say that we understand a group of natural phenomena, we mean that we have found a constructive theory which embraces them."

And for David Hilbert, this included mathematics and its epistemology. Hilbert's Programme (developed roughly over the period 1904-1928) was (to put it simply) to capture mathematics in complete, consistent theories. Hilbert's 'Entscheidungsproblem' asking (again in simple terms) for an algorithm for deciding if a given sentence is logically valid or not - was an important aspect of the programme. We can see it as growing out of Hilbert's view that:

"For the mathematician there is no Ignoramibus, and, in my opinion, not at all for natural science either. ... The true reason why [no one] has succeeded in finding an unsolvable problem is, in my opinion, that there is no unsolvable problem. In contrast to the foolish Ignoramibus, our credo avers:

We must know,

We shall know."

Already, the day before this opening address to the Society of German Scientists and Physicians in Königsberg, September 1930, Gödel had announced, at a different meeting in another part of the same city, the incompleteness result that was to have such negative impact on Hilbert's programme. And in 1936, it turned out that not only could one not fully capture mathematical truth in axiomatic theories but, worse, that which was captured was, in a sense, not captured at all!

Church showed no such algorithm for the Entscheidungsproblem existed. And so did Turing essentially showing:

(1) The set of inputs $n \in \mathbb{N}$ on which $U$ halts is computably enumerable but not computable, and hence:

(2) The logically valid sentences form an incomputable c.e. set.

This was just the beginning. Soon a whole repertoire of incomputable c.e. sets appeared, and any reasonably rich theory turned out to be undecidable. Even questions of interest to 'real' mathematicians were caught in the net. Turing himself played a part in the proving of the unsolvability of the word problem for groups (Post/ Markov 1947, Turing 1950, Novikov/ Boone 1955).

There are two footnotes to the story of Turing's 1936 paper.

We should remember there was a second, 1937 paper, of course. Turing was prone to technical mistakes, and these needed correcting. Gandy ([19], Preface to the 1936-37 papers, p.9) nicely draws together the personal and the scientific again, always important with Turing (my underlining):

"Whether calculating mentally or with pencil and paper, Turing was methodical only by fits and starts, and often made mistakes. [When I came to know him later the 
phrase 'What's a factor of two between friends?' had become a catchword.] But he understood very well what it meant to be totally methodical. Indeed an acceptance - sometimes ready, sometimes reluctant - of the dichotomy between the clearly perceived ideal and the confused actuality was fundamental in Turing's thought."

And it was Alonzo Church who first proved Church's Theorem (as the negative solution to the Entscheidungsproblem came to be called). This time it is Hodges ([21], p.114) who finds the scientific pearl in the Turing personal oyster:

"If he had been a more conventional worker, he would not have attacked the Hilbert problem without having read up all of the available literature, including Church's work. He then might not have been pre-empted - but then, he might never have created the new idea of the logical machine, with its simulation of 'states of mind', which not only closed the Hilbert problem but opened up quite new questions."

\section{BUT INCOMPUTABILITY INTO THE LONG GRASS ...}

Now we come to one of the oddest and most interesting things about the the 1936 discoveries and their repercussions. The emergence of incomputability soon after that of quantum unpredictability, with its iconoclastic import for old certainties, was hardly acknowledged outside the hermetic confines of pure mathematics. At the same time, the universal Turing machine, a mere by-product of that unexpected glimpse beyond Laplacian predictability, became the basis of an informational revolution built on filling out the computable (see, for example, Chapter 7 of [8]).

What happened, and why? Was what happened the natural order of things, or can we see some shape and development underlying these events?

A first observation is that 1936 not only saw a new clarity about what 'incomputability' really is, but was accompanied by the emergence of a conceptual framework which actually took us away from the real world and the uncertainties facing working scientists. Sometimes, being precise about notions has negative as well as positive consequences. It may make them technically more useful, while disrupting their more general usefulness. In the years following 1936 we saw the birth of Recursion Theory, and new notions inimical to vague intuitions.

This involved a dual defeat. Not just that of the outsider trying - not very hard it often seemed! - to understand what recursion theorists were doing, and why, and failing. But of the specialist looking out, usually when seeking funding. One saw a growing emphasis on purely mathematical issues, extending to logic in general. The technical work was often accompanied by an overpowering feeling of reality and subjective significance - but guided by an introjected real world rather than an actual one. A number of those with a professional interest in incomputability (and in large cardinals, for that matter) were even convinced finitists in relation to the material universe. Not for them far-reaching speculations about how the universe, or the human mind, transcends the Turing-barrier, at least not in public. On the other hand, some wonderful mathematics resulted (see Odifreddi's monumental work [25], [26]), and this isolated development, one suspects, was entirely necessary to the future relevance of the subject. Only now are new connections being made (see, for example, [3]), based on the very hardest technical work.

At the same time, there seemed to be a growing belief that mathematics - and science in general - could carry on much as before without ever bumping into incomputable objects. Laplacian predictability was not just a useful model, it was a lasting mind-set amongst working scientists.

There were also mathematical developments which suggested incomputability might be just as containable as sub-atomic unpredictability had become within quantum theory. John Myhill [24] showed that all the unsolvable problems discovered in the 1930s were essentially the same. They were just notational variants of what he called a creative set. One could artificially manufacture other incomputable objects, but there was no evidence of their existence outside of academic 
papers. Perhaps incomputability was not as pervasive and naturally occuring as it had at first seemed...

Anyway, there seemed little point in worrying about the recursion theorists' negative news, when there was so much computability to explore. The richness of the computable universe was increasingly revealed, and provided more than enough work for a whole army of researchers. And there was even a mathematical dimension to this. Reverse mathematics emerged as an attempt to rescue Hilbert's programme - with some success, it seemed to many. When leading proponent Harvey Friedman challenged the recursion theorists to produce just one natural example of an incomputable object (see, for example [17]), he chose his own rules - a peculiarly mathematical definition of "natural" - which left them with nowhere to turn.

\section{THE SEARCH FOR REALLY NATURAL EXAMPLES}

Back in the real world, there is a definition of "natural" understood by everyone. Not a metaphorical one, but one of quotidian usefulness. The New Oxford Dictionary of English (1998 edition), gives it to us as:

"natural - existing in or caused by nature"

Numbers - meaning the natural numbers - are generally thought to qualify here. In fact, the whole of school arithmetic does. In 1972, Yuri Matiyasevich put the finishing touches to a remarkable proof, many years in the making, that the sort of arithmetical questions a school student might ask lead directly to a rich diversity of incomputable sets - a diversity which went far beyond those original examples from the 1930s. Once again, it was David Hilbert being audaciously wrong which provided the impetus, and drew public attention to the result. The negative solution to Hilbert's Tenth Problem, collectively due to Martin Davis, Matiyasevich himself, the philosopher Hilary Putnam, and, crucially, Julia Robinson, demonstrated that all the incomputable sets which could be artificially enumerated by clever logical techniques were not artificial at all - they arose naturally as solution sets of the familiar diophantine equations. It is everyday mathematics leads us unavoidably to incomputable objects.

For the working scientist, naturalness is captured within a rather different kind of mathematics, full of real numbers and differential equations. Marian Pour-El was one of the first to look for appropriate notions and evidence of incomputability arising from this context. One of the most widely-known and enduring discoveries, due to Pour-El and Richards, was a differential equation with computable boundary conditions leading to incomputable solutions. More recently, highprofile names (such as Roger Penrose, Steve Smale) have been associated with investigations of the computability of well-known mathematical objects with known connections with complexity in nature, such as the Mandelbrot and Julia sets.

The closer one gets to physical reality, the more potentially persuasive, while at the same time more speculative and ultimately elusive, the examples become. Leaving aside for the moment the workings of the human brain, the most scrutinised and puzzling part of the physical world is the sub-atomic level. The predictive incompleteness of quantum theory refuses to go away, giving rise to different 'interpretations' which leave us a long way from characterising the algorithmic content of the events it seeks to describe. The quantum process which seems to escape the predictive net most radically, the most promising avatar of incomputability, is by-passed by current quantum computational models - despite recent claims (see Tien Kieu [22]). This is Andrew Hodges' comment on the situation (taken from his article What would Alan Turing have done after 1954?, in Teuscher [36]):

"Von Neumann's axioms distinguished the $\mathbf{U}$ (unitary evolution) and $\mathbf{R}$ (reduction) rules of quantum mechanics. Now, quantum computing so far (in the work of Feynman, Deutsch, Shor, etc) is based on the $\mathbf{U}$ process and so computable. It has not made serious use of the $\mathbf{R}$ process: the unpredictable element that comes in with reduction, measurement, or collapse of the wave function." 
Observable signs of incomputability in nature are not so obvious at the classical level - we are more embroiled and cannot so easily objectify what we are part of. As early as 1970, Georg Kreisel was not deterred, and in a footnote to [23] (p.143) went so far as to propose the possibility of a collision problem related to the 3-body problem which might give "an analog computation of a non-recursive function (by repeating collision experiments sufficiently often)".

This conjecture has come to seem less outrageous as people from various backgrounds have come up with even more basic proposals. One of these comes out of recent progress on the Painlevé Problem, from 1897, asking whether noncollision singularities exist for the $N$-body problem for $N \geq 4$. For $N \geq 5$, Jeff Xia in 1988 showed the answer is "Yes" (see Saari and Xia [33]). And actual, or even uncompleted, infinities in nature are what open the door to incomputability. There are a number of other recent examples.

\section{THE INCOMPUTABLE - SO NEAR BUT SO FAR...}

Turing himself returned to the topic of incomputability in what is usually thought of as a rather opaque 1939 paper.

The background situation established in the years 1931-36 puzzled many people, including Turing himself. They knew that no Turing machine could prove all the true sentences of arithmetic. (Turing's work had enabled a precise notion of formal system to apply Gödel's incompleteness procedure to.) But, it seemed, a human observer could transcend what any given such machine could prove.

An immediate question was: Is there a mathematical analysis throwing light on the apparent ability of the human mind to transcend the mechanical - hopefully based on an extended constructivism? Gödel, in setting out to verify elements of Hilbert's programme, had found his incompleteness theorem. Now Turing, in trying to place the mathematician's thought processes within a constructive framework, was to reveal a similarly surprising scenario. Andrew Hodges ([21], p.137) refers to Turing's conversations with on his way back to America in 1937:

"... now [Turing] gave the impression that he had long been happy with the Russellian view, that at some level the world must evolve in a mechanistic way. ... Symbolically, the Research fountain pen that Mrs Morcom had given him in 1932 was lost on the voyage."

Turing was visiting the Institute of Advanced Study in Princeton, and writing a $\mathrm{PhD}$ thesis under Alonzo Church - one of the few celebrated mathematicians he had hoped to meet there (such as Gödel and von Neumann) who were actually in residence during his time there. It was this thesis which would provide the material for the 1939 paper [38].

Roughly speaking, the key idea - perhaps originating with Church - was to use the constructive ordinals $\mathcal{O}$ of Church and Kleene (1937) to inductively extend theories via Gödel-like unprovable sentences. Turing was specially interested in bringing the true $\Pi_{2}^{0}$ sentences of arithmetic within this constructive framework. He observed that most mathematically interesting problems, such as the Riemann hypothesis, are met at that level.

His partial success was to get a hierarchy containing proofs for all true $\Pi_{1}^{0}$ sentences of arithmetic. Even this outcome was not entirely satisfactory - it turned out that different hierarchies of this kind can be complete (i.e., exhaustive), or invariant (not vary with different notations for the same ordinal), but not both. Turing was interested in invariance, since he saw that as allowing one to unambiguously classify problems according to their 'depth' (i.e., level of ordinal notation). Anyway, the main disappointment was that the techniques did not seem to work for $\Pi_{2}^{0}$ sentences (this result awaited Feferman and the use of stronger reflection principles).

For many people, Turing's 1939 paper is best known for the first appearance there of oracle Turing machines. In investigating the 2-quantifier sentences, Turing sought a constructive derivation of a non- $\Pi_{2}^{0}$ problem - and in so doing invented relativisation (using oracle machines) and, in essence, the Turing jump! 
This led to Post's wonderful 1944 paper [31], and 1948 short abstract [32], which described some first far-reaching consequences (such as Post's theorem, the notion of Turing reducibility, the degrees of unsolvability), and clarified what is happening in the 1939 paper. These three publications of Turing and Post established the still crucial theme of the interrelationship between computability and information content, and established the Turing universe of algorithmically related reals as the standard model for computationally complex environments.

\section{COMPUTING THE INCOMPUTABLE?}

Despite its technical complexities, made even less approachable by being based (presumably at Church's suggestion) on the lambda calculus model of computability, the 1939 paper has a characteristically mundane motivation. Turing claims to clarify here the relationship between 'ingenuity' (subsumed within the ordinal logics) and 'intuition' (needed to identify good ordinal notations - an $\emptyset^{(\omega)}$ level intuition!) Turing clearly regards ingenuity as being what a clever Turing program is capable of, and intuition as something else. There is a clear implication that intuition is a feature of human mental processes, and to that extent Turing is certainly saying that his hierarchies have something to say about how the mathematician's mind transcends his own model of machine computability - even if the results can be subsequently translated into proofs implementable by a Turing machine!

But what about 'hypercomputation'? - often associated nowadays with: Computing the incomputable using oracle Turing machines (where this use may be implicit). The claim is (see, for example, Copeland [4], [5], Copeland and Proudfoot [7]), that Turing anticipated such a possibility, and that oracle Turing machines have been neglected in this respect until rediscovered in the late nineteen-nineties. Well, it takes a truly creative reading of the paper to give oracle machines such an explicit role in Turing's thinking about incomputability, at least at this point. That is not said with the dismissiveness of Martin Davis' recent articles (see, e.g., [9]). It is certainly true - see below - that computers which interact played an important part in Turing's later thinking, and that there was never any evidence that Turing thought the human mind was limited to computing recursive functions. But no - oracle machines, despite their undoubted relevance, are tangential to Turing's thoughts here.

Let us get back to what Turing ([38], pp.134-5), actually says about the underlying meaning of his paper (my underlining):

"Mathematical reasoning may be regarded ... as the exercise of a combination of ....intuition and ingenuity. ... In pre-Gödel times it was thought by some that all the intuitive judgements of mathematics could be replaced by a finite number of ... rules. The necessity for intuition would then be entirely eliminated. In our discussions, however, we have gone to the opposite extreme and eliminated not intuition but ingenuity, and this in spite of the fact that our aim has been in much the same direction."

So he is addressing the familiar mystery of how we often arrive at a mathematical result via what seems like a very unmechanical process, but then promptly retrieve from this a proof which is quite standard and communicable to other mathematicians. Another celebrated mathematician, well-known for his interest in the role of intuition in the mathematician's thinking, was Poincaré. A few years after Turing wrote the above passage, Jacques Hadamard [20] recounts how Poincare got stuck on a problem (the content of which is not important):

\footnotetext{
"At first Poincaré attacked [a problem] vainly for a fortnight, attempting to prove there could not be any such function ... [quoting Poincaré:]

Having reached Coutances, we entered an omnibus to go some place or other. At the moment when I put my foot on the step, the idea came to me, without anything in my former thoughts seeming to have paved the way for it ... I did not verify the idea ...I went on with a conversation already commenced, but I felt a perfect certainty. On my return to Caen, for conscience sake, I verified the result at my leisure."
} 
Who else but Turing would have attempted a mathematical explanation at that time? His argument is still not widely known, and its signficance certainly not understood, except by those at ease with both the mathematics and with thinking about the world in the sort of basic terms which came naturally to Turing.

Emil Post (see [30], p.55) was less subtle in 1941, anticipating others such as Penrose:

“... we may write

\section{The Logical Process is Essentially Creative}

This conclusion, ... makes of the mathematician much more than a kind of clever being who can do quickly what a machine could do ultimately. We see that a machine would never give a complete logic; for once the machine is made we could prove a theorem it does not prove."

By the 1990s this sense of context is lost. By now it is no surprise to find this common amongst those of a recursion theoretic background. Here is a typical quote from Stephen G. Simpson, taken from a widely circulated communication to the Foundations of Mathematics (FOM) e-mail list, August 18 1998:

“... Soare insists ...that computability includes relative computability, i.e. relative recursiveness as a means of classifying non-recursive sets. This terminology strikes me as wrong-headed, as if one were to insist that biology includes the study and classification of inanimate objects."

Here is no longer any sense of engagement, beyond the purely mathematical, with the complexities encountered at the interface between the computable and the incomputable. Robert Soare's call (e.g., [35]) for 'recursion theory' to be returned to the computability-theoretic terminology of Turing is angrily rejected. The mathematics is deeply real to the writer quoted, but this reality is a hermetic one, the science unrecognisable to a latter-day Turing.

This is not to say Turing's 1939 paper did not have a very important technical influence within logic. This was very much the case in regard to proof theory. For instance, Feferman et al were subsequently to replace Turing's consistency principles with much more powerful reflection principles, and (see [15]) much improve on Turing's main results.

In fact, Feferman nicely clarified Turing's paper, including translating proofs out of the lambdatheoretic framework, in his 1988 article [14] Turing in the Land of O(z). And on p.127 of that paper Feferman comments:

" ...Turing anticipated ...the classification by ordinals of the provably (total) recursive functions of various formal systems, obtained later by proof-theoretical work."

One can even find an anticipation of the Paris-Harrington [27] bringing of Gödel's independence result for Peano arithmetic closer to home.

This paper of Turing is a paper packed full of ideas, of course. In section 10 ('The continuum hypothesis. A digression') Turing pursues a set-theoretic analogy, indicating how to replace $\omega_{1}$ by the constructive ordinals, and the subsets of $\omega$ by the computable reals - so anticipating subsequent hierarchies of computable functions.

Later - Feferman's work [13] on 'autonomous' ordinal logic (see Franzén [16] for a readable account) puts Penrose's brave but flawed speculations [28] on computability and the mind in context.

\section{THE TURING UNIVERSE}

Turing's oracle machines give the real numbers an algorithmic infrastructure, which comprises the Turing universe. Emil Post [32] gathered together the computably equivalent reals of this structure, 
and called the resulting ordering the degrees of unsolvability - later called the Turing degrees (poor Post rarely got the credit) - and this has become the mathematical context for the study of the Turing universe. And so arose one of the most forbidding and esoteric research topics in the whole of logic, or mathematics, for that matter.

It is only recently that attention has turned to Turing's universe of computably related reals as providing a model for scientific descriptions of a computationally complex real universe (see [1], [3], [4], etc.) This is based on a growing appreciation of how algorithmic content brings with it implicit infinities, and a science - increasingly coming to terms with chaotic and non-local phenomena - necessarily framed in terms of reals rather than within some discrete or even finite mathematical model. There is a huge amount of research activity, much of it ad hoc in nature, concerned with the computational significance of evolutionary and emergent form, and emergence in more specific contexts. And while purists may claim this is 'nothing to do with logic', there is undoubtedly an urgent need here for the unifying and clarifying role of basic mathematical structures. (As is well-known, Turing himself was very interested in the emergence of form in nature, and wrote seminal papers on the topic - for example [41].)

Of course, the persuasiveness of the full Turing model depends on the extent to which one sees around one avatars of incomputability, and there is a strand of thought - the hypercomputational, as Jack Copeland and others term it - concerned with contriving incomputability via explicitly physical versions of the Turing universe. What is common to both the computability-theoretic and hypercomputational strands is that both the emergence of incomputability, and the emergence of new relations in a universe which admits incomputability, are based on a better understanding of how the local and the global interact. Whatever the context, the key mathematical parallel here is that of definability or invariance, even if within rather different corresponding frameworks. This is not very explicit in building hypercomputational models, which enables Martin Davis and others to trivialise what is happening as being the use of oracles to shuffle around existing incomputability.

Our renewed awareness of real-world relevance puts our view of the pure mathematical theory of the Turing universe in a new light. An area of research which became known for its mathematical unlovelyness and forbidding pathology turns out to be the ideal counterpart to real-world complexity.

This was not at all clear during the recursion theoretic years. The difficulty of the area may have surrounded researchers of the 1960s - pre-eminently Gerald Sacks - with a vaguely heroic, even machismatic, aura. But as time went on this had become a double-edged weapon, and by the 1990s almost no one was impressed by the length and incomprehensibility of groundbreaking new proofs. 'Touching the Void' - and having accidents - was all very well for mountaineers but, as the new century approached, mathematics was very much about deliverables. At times the very value of research into relative computability was questioned. Images of such dissent stick in the memory: Sacks himself, lecturing at Odifreddi's CIME summer school in Bressanone, Italy in 1979, illustrating his view of 'Ordinary Recursion Theory' with a slide of the Chinese masses in cultural revolution turmoil - his metaphor for an activity obsessive, formless, pointless; or, ten years later, Robin Gandy's contribution to a discussion on the future of logic, at a conference in Varna, Bulgaria - communicating an impression of the structure of the Turing degrees via exaggeratedly desperate scribbles on a blackboard.

By the end of the last century the subject would have been unrecognisable to a returning Alan Turing. We had a Turing universe framed by failed mathematical ambitions, and isolated from its natural home, the complexity of the material world. And a reluctance by researchers to stray beyond purely technical questions, unlike Turing himself. The purely mathematical horizons of its brightest minds were focused on an esoteric enterprise centred around something called the bi-interpretability conjecture. The idea was to show that the Turing universe was really just secondorder arithmetic in disguise, a messy mathematical structure, but messy in a mathematically familiar, even respectable, way. A rigid structure, its definable relations easily read off via their counterparts in true second-order arithmetic. Luckily for computability theorists and everyone 
else, this reductionism ran its course, and we still have a complex world in need of the theoretical insights of the mathematical legatees of Alan Turing.

Finally, I should mention that the conceptual leap which gave us the oracle computing machine had wider mathematical consequences. As Sol Feferman says in [14] (Turing in the Land of $\mathrm{O}(\mathrm{z})$, p.127):

"... eventually, the idea of transforming computability from an absolute notion into a relative notion would serve to open up the entire subject of generalized recursion theory."

And that of course is another story, which will have to be told elsewhere.

\section{MIND AND THE CHURCH-TURING THESIS}

Turing himself was interested in the nature of physical computability right to the end. He may not have had in mind specific generalisations of the Church-Turing thesis - see Odifreddi [25] for the best introduction to this topic - but his preoccupation with the links between human and mechanical computability forced him to confront the underlying realities of physical computation. The following quote from Feferman's Turing in the Land of $O(z)$ (see [14], pp.131-2), gives the usual impression people have of Turing's outlook:

"Turing, as is well known, had a mechanistic conception of mind, and that conviction led him to have faith in the possibility of machines exhibiting intelligent behavior."

But these words have been carefully chosen, and if read as carefully do not prevent us following the often shifting balance in Turing's real world between logic and science. At one extreme we have Turing, founder of Artificial Intelligence and seminal influence on its methodology (e.g., via the Turing Test). At the other we have his interest in quantum theory, running through his writings for Mrs Morcom, right up to his late postcards to Robin Gandy (see [21], p. 512). And in between he considered possibilities, coming out of his 1944-48 experiences of the ACE ('Automatic Computing Engine') project, such as machines which make mistakes. Here is Turing in his talk to the London Mathematical Society, February 20, 1947 (quoted in Hodges, p.361):

"... if a machine is expected to be infallible, it cannot also be intelligent. There are several theorems which say almost exactly that."

Turing also anticipated the importance now given to connectionist models of computation. See his discussion of 'unorganised machines' in [39], and Jack Copeland and Diane Proudfoot's article [6] "On Alan Turing's Anticipation of Connectionism".

A 'mechanistic conception of mind' maybe, but no crude extension of the Church-Turing thesis in sight, even at a time when Turing had a huge personal investment in the development of computing machinery. What one sees in Turing's thinking is an attempt to relate computabilitytheoretic structure to physical reality in a very bold and basic way. Maybe he did not mention oracles after 1939, but he never ceased to emphasise the importance of context for real-world computation. The following quote is from the same talk as the previous one (Hodges, p.361 again):

"No man adds very much to the body of knowledge. Why should we expect more of a machine? Putting the same point differently, the machine must be allowed to have contact with human beings in order that it may adapt itself to their standards."

The mysteries Turing grappled with remain. To what extent is the logic of a Turing machine sufficient to capture the workings of a human brain? What is the nature of the mechanical in the physical world? And what relationship does this have to the mind?

He was to make one more major contribution to how we approach, and give meaning to, such difficult questions. 


\section{TURING'S SEMINAL 1950 AI PAPER}

Nowadays one's heart might sink on the reading of an introduction to a paper which proclaimed:

"I propose to consider the question, 'Can machines think ?'”

We have become too used to inflated claims in this area, ones which turn out to repeat old arguments and, more often than not, old confusions. But Turing's 1950 paper [40] on Computing machinery and intelligence, in Mind, both managed to say something new, and, as one expects from Turing, is as careful in formulating the question as it is in giving answers. Despite what some people think, he did not ask "Is the human mind a Turing machine", and still less did he answer, or even imply, "yes" to such a question.

What he did ask ([40], p.442) became the basis for the standard approach to gauging the subsequent success of the $\mathrm{Al}$ enterprise:

The Turing Test: 'Are there imaginable digital computers which would do well in the imitation game?'

Roughly speaking, the imitation game was described in terms of asking a human interrogator to distinguish between a human and a mechanical respondent, within a fixed span of time and under conditions which did not unfairly favour either of them. And in the paper, Turing does confess to a personal opinion. He makes ([40], p.442) a surprisingly limited claim for potential machine intelligence:

"I believe that in about fifty years' time it will be possible to programme computers ... to make them play the imitation game so well that an average interrogator will not have more than 70 per cent. chance of making the right identification after five minutes of questioning. The original question ... I believe to be too meaningless to deserve discussion."

Whether or not Turing had a "mechanistic conception of mind", his background 1938-39 experiences were not forgotten. On the one hand he knew how Gödel's theorem could create an illusion that people transcend computers, and this feeds into Turing's consideration of the 'The Mathematical Objection' to computers doing well at the imitation game. On the other hand, he would remember how his ordinal logics falsely suggest machines transcend the computable and their ultimate failure to 'eliminate' intuition. The discussion is characteristically full of interest. For instance he makes a brave attempt at dealing with an objection based on 'Continuity of the Nervous System'. And, yet again, he mentions 'Learning Machines'. Over the years, oracles continued to feature implicitly in Turing's thinking about machine intelligence. And at no time did he imply that the human mind could be modelled by a standard unaided Turing machine.

While preparing the talk on which this article is based, I wrote to my old friend George ${ }^{2}$ Odifreddi, whose opinion on this point I thought might be useful. This is an extract from what he wrote back to me on May 1st, 2004:

“... when reading turing's 1939 paper i DID have the impression that he thought that by an oracle he meant a human being, and thus that non-computable functions could be humanly computable. the oracle device could be thought of as a formalization of a human-machine interaction, in which the calls to the oracle(s) would be a kind of human help received by the machine. if this interpretation were correct, then it would mean that turing did not accept the church-turing thesis that recursive = human computability. quite the contrary, actually."

I am grateful to George for allowing me to use what is, of course, a hurriedly composed e-mail, although one which I believe to be broadly correct. The extensive literature discussing mechanism, the mind, and the work and opinions of Gödel and Turing, does not get us much closer to a

\footnotetext{
${ }^{2}$ Piergiorgio
} 
definitive view. Two recommendable recent contributions treading similar ground with differing outcomes are Shapiro [34] and Piccinini [29].

\section{THE TURING RENAISSANCE}

What is currently so exciting is that the sorts of questions which preoccupied Turing, and the very basic extra-disciplinary thinking which he brought to the area, are being revisited and renewed by researchers from quite diverse backgrounds. What we are seeing is an emergent coming together of logicians, computer scientists, theoretical physicists, people from the life sciences, and the humanities and beyond, around an intellectually coherent set of computability-related problems. The recurring and closely linked themes here are the relationship between the local and the global, the nature of the physical world, and within that the human mind, as a computing instrument, and our expanding concept of what may be practically computable.

The specific form in which these themes become manifest are quite varied. For some there is a direct interest in incomputability in Nature, such as that coming out of the $n$-body problem or quantum phenomena. For others it is through addressing problems computing with reals and with scientific computing. The possibility of computations 'beyond the Turing barrier' leads to the study of analog computers, while theoretical models of hypercomputation figure in heated crossdisciplinary controversies. There is also intensive research going on into a number of practical models of natural computing, which present new paradigms of computing whose exact content is as yet not fully understood. In many scientific areas the emergence of form is deeply puzzling, and there is a need for mathematical models. Turing played an anticipatory role here too (Odifreddi tells "[Gerald] Edelman quotes Turing as a precursor of his work on morphogenesis").

What is going on here is fascinating. What is taking shape seems to be the 1936 paradigm shift renewed. In the 1920s and 1930s we saw a fracturing of the comfortable picture of how science could bring predictability to a complex universe, unaccompanied by any overall concept of the underlying mathematical structures. We now see a coming together (at times faltering, at times confused) of science and mathematics to replace the Laplacian model of science with one whose complexities match those of the real world. At the root of this is both the technical legacy of Turing, and the kind of unified approach to scientific problems that is so characteristic of Turing's own thinking.

The role of the classical computability theorist here is, as yet, peripheral. There is little awareness and understanding of much recent research directly descended from Turing's work on incomputability and his notion of relative computability, beyond the tiny community of specialists. (Turing's computability-theoretic heirs are significantly absent from Teuscher's recent book [36] otherwise such a useful window on this Turing rennaissance.) Much of the work being done has a mathematically naive aspect on which sceptics such as Martin Davis have been quick to pounce. But the separation since Turing - during the recursion theoretic era - between computability theory and its real-world counterpart, is being slowly repaired, as is a lingering distrust of current outcomes, on both sides of the divide.

\section{TURING'S FINAL THOUGHTS ON THE MIND AS MACHINE?}

So Alan Turing turns out to be a pivotal figure in a scientific revolution which has not yet run its course. And right to the end he was grappling with the complexities of physical computability and its mathematical models. For Turing the scientist, the human mind was his laboratory. And, as I have suggested, his 1939 paper provided the theoretical framework for all his subsequent thinking on the mind as computer. But a measure of the extent to which he was prepared to engage with everyday problems is the way he was able to communicate his science to everyday people. Here is the final paragraph of Alan Turing's article [42] on Solvable and Unsolvable Problems, in the popular Penguin Science News back in his final year of 1954, (number 31, p.23):

"The results which have been described in this article are mainly of a negative character, setting certain bounds to what we can hope to achieve purely by reasoning. 
These, and some other results of mathematical logic may be regarded as going some way towards a demonstration, within mathematics itself, of the inadequacy of 'reason' unsupported by common sense." (my underlining)

After my talk on which this article is based, Jack Copeland asked me whether I thought that Turing meant "intuition" when he referred here to "common sense" - "if so, I agree". Yes, despite Turing's characteristically cautious phraseology, the above quotation clearly comes from the inventor and interpreter of ordinal logics. Certainly not someone who was content with any existing algorithmic model of the human mind.

And in his final months he was renewing his interest in the parallel mysteries of quantum theory, as indicated by his cryptic Messages from the Unseen World postcards to Robin Gandy (see [21], p.512). Alan was still the writer to Mrs Morcom of those youthful speculations on the links between incomputability in the mind and at the quantum level. Here is Robin Gandy in a letter to Max Newman in June 1954:

"During this spring [Turing] spent some time inventing a new quantum mechanics ... he produced a slogan 'Description must be non-linear, prediction must be linear'.'

\section{REFERENCES}

[1] Cooper S. B. (1999) Clockwork or Turing U/universe? - remarks on causal determinism and computability. In Models and Computability (S. B. Cooper and J. K. Truss, eds.), London Mathematical Society Lecture Note Series 259, Cambridge University Press, Cambridge, pp. 63-116.

[2] Cooper S. B. (2004) Computability Theory. Chapman \& Hall/ CRC Press, Boca Raton, FL, New York, London.

[3] Cooper S. B. and Odifreddi P. (2003) Incomputability in Nature. In Computability and Models: Perspectives East and West (Cooper S. B. and Goncharov S. S., eds.), Kluwer Academic/ Plenum Publishers, New York, Boston, Dordrecht, London, Moscow, pp. 137-160.

[4] Copeland J. (1998) Turing's O-machines, Penrose, Searle, and the brain. Analysis, 58, 12838.

[5] Copeland J. (2000) Narrow versus wide mechanism: Including a re-examination of Turing's views on the mind-machine issue. J. of Phil. 96, 5-32.

[6] Copeland J. and Proudfoot D. (1996) On Alan Turing's anticipation of connectionism. Synthese, 108, 361-377. Reprinted in Artificial Intelligence: Critical Concepts in Cognitive Science (Chrisley R., ed), Volume 2: Symbolic Al. Routledge, London, 2000.

[7] Copeland J. and Proudfoot D. (1996) Alan Turing's forgotten ideas in computer science. Scientific American, 253:4, 98-103.

[8] Davis M. (2000) The Universal Computer: The Road from Leibniz to Turing. W. W. Norton, New York.

[9] Davis M. (2004) The myth of hypercomputation. In Teuscher, pp. 195-211.

[10] Dawson J. W. (1997) Logical Dilemmas: The Life and Work of Kurt Gödel. A K Peters, Wellesley, Mass.

[11] Eddington A. S. (1928) The Nature of the Physical World. Cambridge University Press, Cambridge.

[12] Einstein A. (1950) Out of My Later Years. Philosophical Library, New York.

[13] Feferman S. (1962) Transfinite recursive progressions of axiomatic theories. J. of Symbolic Logic 27, 259-316.

[14] Feferman S. (1988) Turing in the Land of $O(z)$. In The Universal Turing Machine: A HalfCentury Survey (Herken R., ed.), Oxford University Press, New York, pp. 113-147.

[15] Feferman S. (1991) Reflecting on incompleteness. J. of Symbolic Logic 56, 1-49.

[16] Franzen T. (2004) Inexhaustibility - A Non-Exhaustive Treatment. Association for Symbolic Logic/ A K Peters, Wellesley, Mass. 
[17] Friedman H. (1999) FOM message of Mon., 2 Aug. 1999. Foundations of Mathematics Archive, www.cs.nyu.edu/pipermail/fom/1999-August/003326.html.

[18] Gandy R. O. (1988) The confluence of ideas in 1936. In The Universal Turing Machine: A Half-Century Survey (Herken R., ed.), Oxford University Press, New York, pp. 51-102.

[19] Gandy R. O. and Yates C. E. M. eds. (2001) Collected Works of A.M. Turing: Mathematical Logic. North-Holland, Amsterdam, New York, Oxford.

[20] Hadamard J. (1945) The Psychology of Invention in the Mathematical Field. Princeton Univ. Press, Princeton.

[21] Hodges A. (1992) Alan Turing: The Enigma. Vintage, London, Melbourne, Johannesburg.

[22] Kieu T. (2003) Quantum algorithm for the Hilbert's Tenth Problem. Int. J. Theoretical Physics 42, 1461-1478.

[23] Kreisel G. (1970) Church's Thesis: a kind of reducibility axiom for constructive mathematics. In Intuitionism and Proof Theory: Proceedings of the Summer Conference at Buffalo N.Y. 1968 (Kino A., Myhill J., and Vesley R. E., eds.), North-Holland, Amsterdam, London, pp. 121-150.

[24] Myhill J. (1955) Creative sets. Z. Math. Logik Grundlag. Math. 1, 97-108.

[25] Odifreddi P. (1989) Classical Recursion Theory. North-Holland, Amsterdam, New York, Oxford, Tokyo.

[26] Odifreddi P. (1999) Classical Recursion Theory, Vol. II. North-Holland, Amsterdam, Lausanne, New York, Oxford, Shannon, Singapore, Tokyo.

[27] Paris J. and Harrington L. (1977) A mathematical incompleteness in Peano arithmetic. In Handbook of Mathematical Logic (Barwise J., ed.), North-Holland, Amsterdam, pp. 11331142.

[28] Penrose R. (1994) Shadows of the Mind: A Search for the Missing Science of Consciousness. Oxford University Press, Oxford.

[29] Piccinini G. (2003) Alan Turing and the mathematical objection. Mind and Machines 13, 2348.

[30] Post E. L. (1941) Absolutely unsolvable problems and relatively undecidable propositions Account of an anticipation. In Collected Works of Post (ed. Davis M.), 1994, pp.375-441.

[31] Post E. L. (1944) Recursively enumerable sets of positive integers and their decision problems. Bull. of the Amer. Math. Soc. 50, 284-316.

[32] Post E. L. (1948) Degrees of recursive unsolvability: preliminary report. (abstract). Bull. Amer. Math. Soc. 54, 641-642.

[33] Saari D. G. and Xia Z. (Jeff) (1995) Off to infinity in finite time. Notices of the Amer. Math. Soc. 42, 538-546.

[34] Shapiro S. (1998) Incompleteness, mechanism, and optimism. Bull. of Symbolic Logic 4, 273-302.

[35] Soare, R. I. (1996) Computability and recursion. Bull. of Symbolic Logic 2, 284-321.

[36] Teuscher C. (ed.) (2004) Alan Turing: Life and legacy of a great thinker. Springer-Verlag, Berlin, Heidelberg.

[37] Turing A. M. (1936) On computable numbers, with an application to the Entscheidungsproblem. Proc. London Math. Soc. (2) 42 (1936-7), pp. 230-265. Reprinted in A. M. Turing, Collected Works: Mathematical Logic, pp. 18-53.

[38] Turing A. M. (1939) Systems of logic based on ordinals. Proc. London Math. Soc. (2) 45, pp. 161-228. Reprinted in A. M. Turing, Collected Works: Mathematical Logic, pp. 81-148.

[39] Turing A. M. (1948) Intelligent machinery. National Physical Laboratory Report. In Machine Intelligence 5 (Meltzer B. and Michie D., eds.), Edinburgh University Press, Edinburgh, 1969, pp. 3-23. Reprinted in A. M. Turing, Collected Works: Mechanical Intelligence (Ince D. C., ed.), North-Holland, Amsterdam, New York, Oxford, Tokyo, 1992.

[40] Turing A. M. (1950) Computing machinery and intelligence. Mind 59, 433-460. Reprinted in A. M. Turing, Collected Works: Mechanical Intelligence (Ince D. C., ed.), North-Holland, Amsterdam, New York, Oxford, Tokyo, 1992.

[41] Turing A. M. (1952) The chemical basis of morphogenesis. Phil. Trans. of the Royal Society of London, B 237, 37-72. Reprinted in A. M. Turing, Collected Works: Morphogenesis (Saunders P. T., ed.), North-Holland, Amsterdam, New York, Oxford, Tokyo, 1992.

[42] Turing A. M. (1954) Solvable and Unsolvable Problems. Penguin Science News 31, 7-23. 
[43] Turing A. M. (2001) Collected Works: Mathematical Logic (Gandy R. O. and Yates C. E. M., eds.), North-Holland, Amsterdam, New York, Oxford, Tokyo.

[44] von Neumann J. (1951) The general and logical theory of automata. In Cerebral Mechanisms in Behaviour (Jeffress L. A., ed.), John Wiley, New York, pp. 1-41.

[45] Wang H. (1987) Reflections on Kurt Gödel. MIT Press, Cambridge, MA. 